\title{
Metal-organic Frameworks/Graphene Oxide Composites: Preparation and Applications in Dye Absorption
}

\author{
ZHANG Yi ${ }^{\mathrm{a}}$, TAO Cheng-an ${ }^{*}, \mathrm{~b}$, Jia Song ${ }^{\mathrm{c}}$ and Jianfang Wang ${ }^{*, \mathrm{~d}}$ \\ ${ }^{1}$ College of Science, National University of Defense Technology, Changsha, 410073, P. R. China \\ a995056680@qq.com, btaochengan@gmail.com, 'songjia@163.cm, dwangjianfang@nudt.edu.cn
}

\section{Keywords: Metal-organic frameworks; Graphene; Dye; Wastewater; Porous materials}

Abstract. Metal-organic frameworks (MIL-100)/graphene oxide composites (MOFs/GO) with different graphene oxide (GO) contents were prepared under hydrothermal conditions. The resultant products were then characterized by powder X-ray diffraction, infrared spectra, thermogravimetric analysis and nitrogen adsorption desorption. Methyl orange and methyl red solutions were used as simulative dye wastewater to evaluate the adsorption performance of resulted MOFs/GO composites. The results show that adsorption rate and saturated adsorption amount of dye are affected by the contents of GO in the composites and will be improved simultaneously by adding appropriate amount of GO.

\section{Introduction}

Wastewater from dye industry has become the main pollution source worldwide. Because dyes are of strong affinity to substrate and extreme difficulty in decomposing, this kind of wastewater will cause heavy burden and irreversible destruction to local eco-system. To tackle this problem, lots of technologies and methods, such as, adsorption method, flocculence, photocatalytic degradation etc. have been exploited. Among these methods, adsorption method is one of the most commonly used and the most effectively process, which use porous material with high specific surface area as adsorbent to adsorb and separate dye from wastewater. Traditional porous materials include activated carbon, silicon, zeolite and so on ${ }^{[1,2]}$.

Metal-organic frameworks (MOFs) is a kind of new porous crystalline material combined with metal ions and organic ligand. Due to its immense specific surface area, adjustable pore diameter and structure, MOFs is widely used in gas adsorption ${ }^{[3]}$, separation $^{[4]}$, catalysis ${ }^{[5]}$, sensing ${ }^{[6]}$ etc. And it also showed encouraging result when applied in wastewater treatment ${ }^{[7,8]}$. However, the adsorption rate is relatively low which is limited by its small pore diameter (mircopore magnitude).

Graphene oxide (GO) is derived from graphene which has hydroxyl, carboxyl, epoxy and other oxygen-containing groups while maintain plane structure of graphene. When introduced to MOFs structure, GO forms MOFs/GO composite with MOFs. Consequently, molecule retention will increase with the increasing of disperse force resulted by structure change in MOFs ${ }^{[9]}$.

To fully exploit the adsorption character, we focus our research on MIL-100 whose metal center is ferric ion and ligand is 1, 3, 5-benzenetricarboxylate. GO is prepared by Modified Hummers' Method and the composite is prepared with different ratio of GO and MIL-100 under hydrothermal condition. The sample then is characterized by X-ray diffraction (XRD), infrared spectra (IR), thermogravimetric analysis (TGA) and nitrogen adsorption desorption. In the laboratory condition, Methyl orange (MO) and methyl red (MR) solutions (Figure 1) were used as simulative dye wastewater to evaluate the adsorption performance of resulted MOFs/GO composites. It's found that adsorption rate and saturated adsorption amount of dye are affected by the contents of GO in the composites and will be improved simultaneously by adding appropriate amount of GO.

\section{Experimental section}

Preparation of MIL-100 and MOFs/GO composite. Iron powder (99.9\%), BTC, hydrofluoric acid $(48 \%)$, nitric acid (65\%) and deioned water was added to a Teflon autoclave at a mole ratio of 
1:0.67:2:0.6:277 and kept at $160^{\circ} \mathrm{C}$ for $8 \mathrm{~h}$. The resultant solution was filtrated and the residue was dispersed in $150 \mathrm{~mL}$ deioned water and stirred for $5 \mathrm{~h}$ at $70^{\circ} \mathrm{C}$ and in $\mathrm{EtOH}$ for $3 \mathrm{~h}$ at $60^{\circ} \mathrm{C}$ to activate the product. Finally, the resultant product was dried at $75^{\circ} \mathrm{C}$ to obtain MIL-100 powder, labeled MOFO.

The GO used in this experiment was synthesized from natural graphene powder by a modified Hummers method ${ }^{[11]}$ and characterized as described in our previous works ${ }^{[12]}$. The MOFs/GO composite was prepared the same way except that different ratio of GO was added to the precursor solution. The composites which contains $0.5,2.5,5 \mathrm{wt} \%$ GO were referred as MOF1, MOF2, MOF3 respectively.

Adsorption experiment. 10mg MOFs/GO composite was added to $10 \mathrm{~mL}$ of $\mathrm{MO}$ solution $(10 \mathrm{mg} / \mathrm{L})$ or $\mathrm{MR}$ solution $(20 \mathrm{mg} / \mathrm{L})$ and $0.4 \mathrm{~mL}$ dispersion was taken out at a time for UV/Vis measurement at an interval of 30min. To measure its absorbance, the dispersion was centrifuged and $0.2 \mathrm{~mL}$ supernate was taken and diluted to $2 \mathrm{~mL}$.

\section{Results and Discussion}

Characterization of MOF/GO composites. XRD patterns of the composites are shown in Fig.1A. The position and intensity of diffraction peak is in accord with the result simulated from the crystallographic information file (CIF) of MIL-100(Fe) in previous reports ${ }^{[10]}$. Compared with that of MOF0 which has no GO inside, the curves of MOF1, MOF2, and MOF3 has no evident change, suggesting that the structure of MOF is retained.

(A)

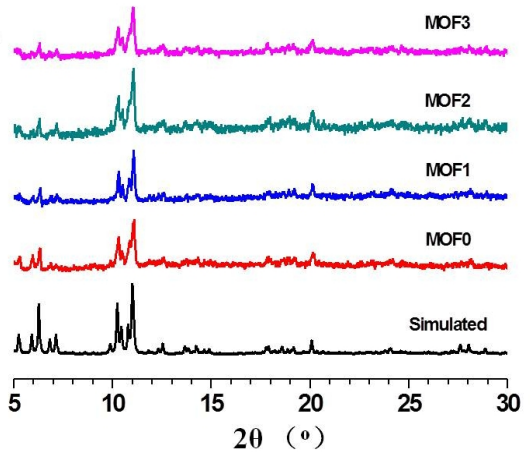

(C)

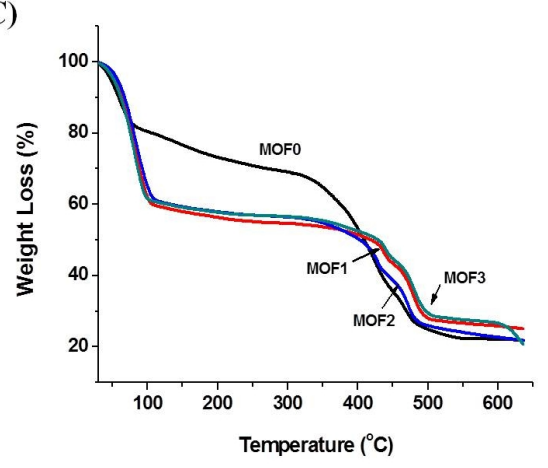

(B)

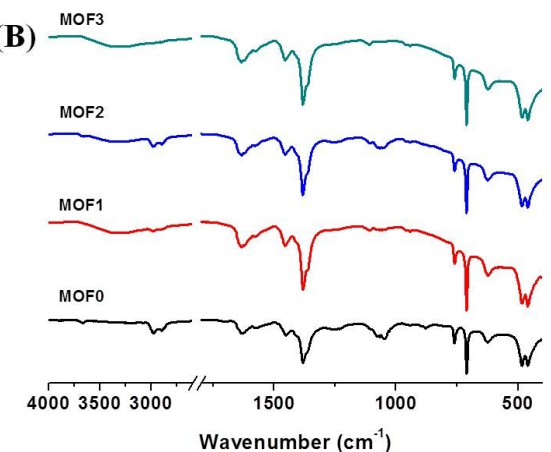

(D)

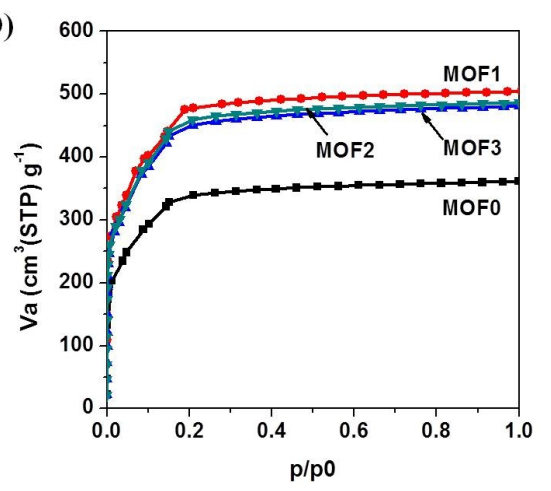

Fig. 1 (A) XRD patterns, (B) FT-IR spectra, (C) TGA curves and (D) N2 adsorption-desorption isotherms of MOFs/GO composites

FT-IR spectra of MOF0 (Fig.1B) reveals that the peak at $1705 \mathrm{~cm}^{-1}$, which is the characteristic peak of carboxyl, is inconspicuous, which exclude the existence of free carboxyl, i.e. All carboxyl groups of BTC have bonded with $\mathrm{Fe}^{3+}$ ions. Compared with MOF0, MOF1, 2, 3 showed no notable differences, which means the concentration of GO in the composite is quite low, and excess of BTC has been washed out. 
TGA curves were shown in Fig. $1 \mathrm{C}, \mathrm{MOF} 0$ contains the least amount of solvent (herein, $\mathrm{H}_{2} \mathrm{O}$ molecule) at about $20 \mathrm{wt} \%$ and is relatively easy to evacuate. And it decomposes at $350^{\circ} \mathrm{C}$. While those composites, which exhibit better stability, decompose at $400^{\circ} \mathrm{C}$, higher than MOF0. Moreover, because of the influence of GO, the composites contain $40 \%$ solvent and evacuate at $100^{\circ} \mathrm{C}$, that is to say, they are more inclined to adsorb solvent molecule, and exhibit stronger retention ability.

Table 1 Specific surface areas and total pore volumes of MOF/GO composites

\begin{tabular}{ccc}
\hline Sample & Specific surface area $(\mathrm{m} 2 / \mathrm{g})$ & Total pore volume $(\mathrm{cm} 3 / \mathrm{g})$ \\
\hline MOF0 & 1279.9 & 0.5590 \\
MOF1 & 1754.0 & 0.7801 \\
MOF2 & 1694.1 & 0.7448 \\
MOF3 & 1725.4 & 0.7530 \\
\hline
\end{tabular}

Fig. 1D shows the $\mathrm{N}_{2}$ adsorption-desorption isotherms of MOFs/GO composites, where MOF0 possesses conspicuous isotherms character of micro-porous materials, indicating that MIL-100 is successfully synthesized. To better compared the pore structures of various composites, the specific surface area and total pore volume of these samples were listed in table 1, which shows the BET specific surface area and pore volume of MOF0 is $1279.9 \mathrm{~m}^{2} / \mathrm{g}$ and $0.5590 \mathrm{~cm}^{3} / \square \mathrm{g}$ respectively, close to what is reported before ${ }^{[13]}$. With the addition of GO, the specific surface area and pore volume increase remarkably. To be more precise, MOF1 increases $37 \%$ in specific surface area and $40 \%$ in pore volume, respectively. Accordingly, MOF2 increases 32\%, 33\% and MOF3 increases 35\%, 35\% respectively. New pores can form as the addition of GO, a proposal for which there is ample precedents reported $[9,11,14,15]$.

Adsorption performance. Fig. 2 shows the adsorption plots of MO. The rate of adsorption of MOF0 is quite high, adsorption rate can reach $25 \%$ in $60 \mathrm{~min}$, and the saturated adsorption rate is about $54 \%$, while MOF1 possess a relatively low rate of adsorption, and in $60 \mathrm{~min}$, it only adsorb less than $10 \%$. However, it nearly has the same saturated adsorption rate as MOF0. The decreased rate may be result of MOF structure is wrapped by GO sheets, making it harder for MO molecule to get in, but this will not affect the saturated amount. Compared with MOF0, MOF2 witnessed an obvious increase in the rate of adsorption and adsorption capacity. The adsorption rate reaches $25 \%$ in 35 min, higher than that of other composites, and the saturated adsorption amount is over $60 \%$, which is more than pristine MOF0.

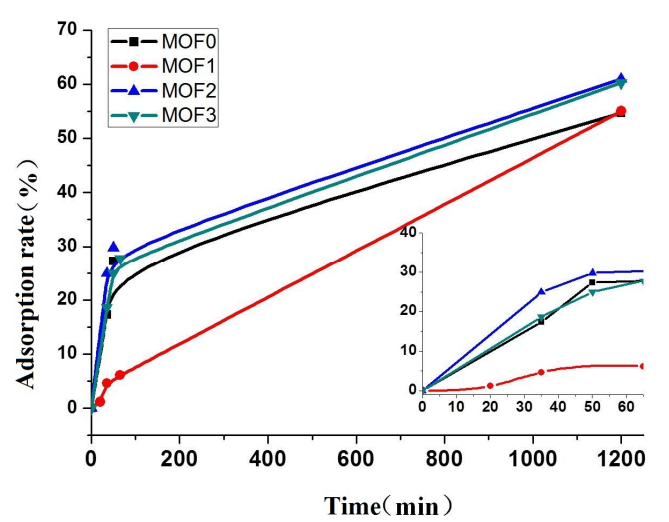

Fig.2 Plots of amounts of MO absorbed by MOFs/GO composites

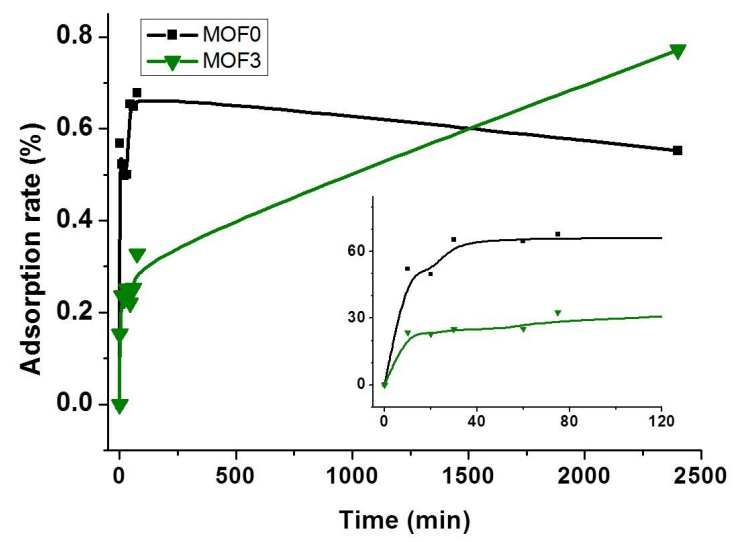

Fig. 3 Plots of amounts of MR absorbed by MOFs/GO composites

MR adsorption plot is shown in Fig.3. Similar to that of MO, MOF0 exhibits a high rate of adsorption, and the adsorption rate can reach $60 \%$ in only 30 min while MOF3 just reach $22 \%$. However, the saturated adsorption amount of MOF3 can reach $77 \%$ while MOF0 experience a slightly decrease after $40 \mathrm{~h}$, finally reaching 55\%. The adsorption activity indicates that GO has stronger 
affinity to dye molecule, therefore the composite has higher stability and saturated adsorption amount, although it will decrease the rate of adsorption because of the weak permeability of GO.

\section{Conclusions}

MIL-100 and its composite with GO was synthesized under hydrothermal condition and characterized by XRD, FT-IR, TGA and $\mathrm{N}_{2}$ adsorption-desorption. The results show that small amount of GO will increase the pore volume and specific surface area while maintaining MOFs' structure. When applied to wastewater treatment, the composite containing $5 \%$ of GO displayed the best performance in saturated adsorption capacity, making it a promising kind of wastewater treatment material. However, because of the influence of MOF structure and weak permeability of GO, composites may experience lower adsorption rate in some cases, but the saturated adsorption amount will increase remarkably, because GO will increase the specific surface area, pore volume, stability of MOFs and the affinity with dyes.

\section{Acknowledgements}

The authors would like to thank the financial support of National Natural Science Foundation of China $(21203247,21573285,61376125)$.

\section{References}

[1] V. K. Gupta, Suhas: J. Environ. Manage. Vol. 90 (2009), p. 2313.

[2] M. A. Montgomery and Elimelech M: Environ. Sci. Technol. Vol. 41 (2007), p. 17.

[3] L. J. Murray, M. Dinca and Long J. R. H: Chem. Soc. Rev. Vol. 38 (2009), p. 1294.

[4] J. R. Li, R. J. Kuppler and Zhou H. C: Chem. Soc. Rev. Vol. 38 (2009), p. 1477.

[5] J. Lee, O. K. Farha, J. Roberts, K. A. Scheidt, S. T. Nguyen and Hupp J. T: Chem. Soc. Rev. Vol. 38 (2009), p. 1450.

[6] A. U. Czaja, N. Trukhan and Muller U: Chem. Soc. Rev. Vol. 38 (2009), p. 1284.

[7] E. Haque, J. E. Lee and Jang I. T: J. Hazard. Mater. Vol. 181 (2010), p. 535.

[8] E. Haque, J. W. Jun and Jhung S. H: J. Hazard. Mater.Vol. 185(2011), p. 507.

[9] Z. Huang, G. Liu and Kang F: ACS Appl. Mater. Interfaces Vol. 4 (2012), p. 4942.

[10] H. Patricia, S. Suzy, S. Christian, H. Do-Young, S. You-Kyong, C. Jong-San, G. Jean-Marc, M. Irene and Gerard F: Chem. Commun. (2007), p. 2820.

[11] D. C. Marcano, D. V. Kosynkin, J. M. Berlin, A. Sinitskii, Z. Sun, A. Slesarev, L. B. Alemany, W. Lu and J. M. Tour: ACS Nano Vol. 4 (2010), p. 4806.

[12] C. Tao, J. Wang, S. Qin, Y. Lv, Y. Long, H. Zhu and Jiang Z: J. Mater. Chem. Vol. 22 (2012), p. 24856.

[13] Y. K. Seo, J. W. Yoon, J. S. Lee, U. H. Lee, Y. K. Hwang, C. H. Jun, H. Patricia, C. Serre and Chang J. S: Microp. Mesop. Mater. Vol. 157 (2012), p. 137.

[14] J. B. Teresa and Camille P: Adsorption Vol. 17 (2011), p. 5.

[15] P. Camille and Teresa J. B: Dalton Trans. Vol. 41 (2012), p. 4027. 